Volume 133, Number 9, Pages 2559-2567

S 0002-9939(05)07976-1

Article electronically published on April 8, 2005

\title{
THE CLASSICAL MONOTONE CONVERGENCE THEOREM OF BEPPO LEVI FAILS IN NONCOMMUTATIVE $L_{2}$-SPACES
}

\author{
BARTHÉLEMY LE GAC AND FERENC MÓRICZ
}

(Communicated by Jonathan M. Borwein)

\begin{abstract}
Let $H$ be a complex Hilbert space and let $\mathfrak{A}$ be a von Neumann algebra over $H$ equipped with a faithful, normal state $\phi$. Then $\mathfrak{A}$ is a prehilbert space with respect to the inner product $\langle A \mid B\rangle:=\phi\left(B^{*} A\right)$, whose completion $L_{2}=L_{2}(\mathfrak{A}, \phi)$ is given by the Gelfand-Naimark-Segal representation theorem, according to which there exist a one-to-one $*$-homomorphism $\pi$ of $\mathfrak{A}$ into the algebra $\mathcal{L}\left(L_{2}\right)$ of all bounded linear operators acting on $L_{2}$ and a cyclic, separating vector $\omega \in L_{2}$ such that $\phi(A)=(\pi(A) \omega \mid \omega)$ for all $A \in \mathfrak{A}$. Given any separable Hilbert space $H$, we construct a faithful, normal state $\phi$ on $\mathcal{L}(H)$ and an increasing sequence $\left(A_{n}: n \geq 1\right)$ of positive operators acting on $H$ such that $\left(\phi\left(A_{n}^{2}\right): n \geq 1\right)$ is bounded, but $\left(\pi\left(A_{n}\right) \omega: n \geq 1\right)$ fails to converge both bundlewise and in $L_{2}$-norm. We also present an example of an increasing sequence of positive operators which has a subsequence converging both bundlewise and in $L_{2}$-norm, but the whole sequence fails to converge in either sense. Finally, we observe that our results are linked to a previous one by R. V. Kadison.
\end{abstract}

\section{BACKGROUND}

Let $H$ be a (separable) complex Hilbert space, $\mathcal{L}(H)$ the algebra of all bounded linear operators acting on $H, \mathfrak{A}$ a von Neumann algebra in $\mathcal{L}(H)$, and $\phi$ a faithful, normal state acting on $\mathfrak{A}$. Then

$$
\langle A \mid B\rangle:=\phi\left(B^{*} A\right), \quad A, B \in \mathfrak{A},
$$

defines an inner product on $\mathfrak{A}$, and $(\mathfrak{A},\langle\cdot \mid \cdot\rangle)$ is a complex prehilbert space. Denote by $L_{2}=L_{2}(\mathfrak{A}, \phi)$ its completion, by $(\cdot \mid \cdot)$ the inner product and by $\|\cdot\|$ the norm in $L_{2}$.

According to the Gelfand-Naimark-Segal representation theorem, there exist a one-to-one $*$-homomorphism $\pi$ of $\mathfrak{A}$ into the algebra $\mathcal{L}\left(L_{2}\right)$ and a cyclic, separating vector $\omega$ in $L_{2}$ such that

$$
\phi(A)=(\pi(A) \omega \mid \omega),
$$

Received by the editors September 2, 2002.

2000 Mathematics Subject Classification. Primary 46L53, 46L10.

Key words and phrases. von Neumann algebra $\mathfrak{A}$, faithful and normal state $\phi$, completion $L_{2}=L_{2}(\mathfrak{A}, \phi)$, Gelfand-Naimark-Segal representation theorem, bundle convergence, classical monotone convergence theorem of Beppo Levi, increasing sequence of positive operators.

This research was started while the second-named author visited the "Centre de Mathématiques et Informatique, Université de Provence, Marseille" during the summer of 2002; it was also partially supported by the Hungarian National Foundation for Scientific Research under Grants T 044782 and T 046192. 
in particular,

$$
\phi\left(A^{*} A\right)=\|\pi(A) \omega\|^{2}, \quad A \in \mathfrak{A} .
$$

In 1996 Hensz, Jajte and Paszkiewicz [2] introduced the notion of bundle convergence for sequences of operators in $\mathfrak{A}$ as well as for sequences of vectors in $L_{2}$. To present its definition, we consider a sequence $\left(E_{k}\right)$ of operators in $\mathfrak{A}_{+}$, the cone of all positive operators in $\mathfrak{A}$, such that

$$
\sum_{k=1}^{\infty} \phi\left(E_{k}\right)<\infty
$$

and associate with it a so-called bundle $\mathcal{P}=\mathcal{P}\left(E_{k}\right)$ defined as follows:

$$
\begin{gathered}
\mathcal{P}:=\left\{P \in \operatorname{Proj} \mathfrak{A}: \sup \left\{\left\|P\left(\sum_{k=1}^{n} E_{k}\right) P\right\|_{\infty}: n \geq 1\right\}<\infty\right. \\
\text { and } \left.\left\|P E_{n} P\right\|_{\infty} \rightarrow 0 \text { as } n \rightarrow \infty\right\} .
\end{gathered}
$$

Here $\operatorname{Proj} \mathfrak{A}$ is the class of all (selfadjoint) projections $P$ in $\mathfrak{A}$, and $\|\cdot\|_{\infty}$ is the usual operator norm.

It is a crucial fact that for any sequence $\left(E_{k}\right)$ in $\mathfrak{A}_{+}$with property (1.2), the bundle defined in (1.3) is "abundant" enough in the sense that it contains projections $P$ arbitrarily close to the identity, operator 1 in $\mathfrak{A}$. In other words, for every $\varepsilon>0$ there exists $P \in \mathcal{P}\left(E_{k}\right)$ such that $\phi(1-P)<\varepsilon$. (See the details in [2].)

Now, a sequence $\left(A_{n}\right)$ of operators in $\mathfrak{A}$ is said to be bundle convergent to some $A$ in $\mathfrak{A}$, in sign: $A_{n} \stackrel{b, \mathfrak{A}}{\longrightarrow} A$ as $n \rightarrow \infty$, if there exists a bundle $\mathcal{P}$ such that for each $P$ in $\mathcal{P}$ we have $\left\|\left(A_{n}-A\right) P\right\|_{\infty} \rightarrow 0$ as $n \rightarrow \infty$. Furthermore, a sequence $\left(\xi_{n}\right)$ of vectors in $L_{2}$ is said to be bundle convergent to some $\xi$ in $L_{2}$, in sign: $\xi_{n} \stackrel{b}{\rightarrow} \xi$ as $n \rightarrow \infty$, if there exists a sequence $\left(A_{n}\right)$ of operators in $\mathfrak{A}$ for which

$$
A_{n} \stackrel{b, \mathfrak{A}}{\longrightarrow} O \text { as } n \rightarrow \infty \quad \text { and } \quad \sum_{n=1}^{\infty}\left\|\xi_{n}-\xi-\pi\left(A_{n}\right) \omega\right\|^{2}<\infty,
$$

where $O$ is the zero operator in $\mathfrak{A}$.

Bundle convergence is an appropriate substitute for almost everywhere (in measure theory) or almost sure (in probability theory) convergence in a noncommutative setting. In addition, it enjoys the property of additivity.

As to details of von Neumann algebras, we refer the reader to the basic books [1] by Dixmier and [8] by Takesaki. (See also [3, pp. 106-138] by Jajte for a rapid exposition.)

\section{MAIN RESUlts}

Let $(X, \mathcal{F}, \mu)$ be a measure space with positive measure $\mu$. The classical monotone convergence theorem of Beppo Levi formulated in terms of functions of the Lebesgue space $L_{2}(X, \mathcal{F}, \mu)$ reads as follows: If $\left(f_{n}: n \geq 1\right)$ is an increasing sequence (that is, $f_{n}(x) \leq f_{n+1}(x)$ for every $n$ and almost every $x$ in $X$ ) of nonnegative, square integrable functions on $X$ such that

$$
\sup _{n \geq 1} \int_{X} f_{n}^{2} d \mu<\infty
$$


then $f_{n}$ converges to some square integrable function $f$ both almost everywhere and in $L_{2}$-norm as $n \rightarrow \infty$.

The following problem arises naturally: Is it possible to get a counterpart of this theorem in the noncommutative setting?

Problem 1. Let $\left(A_{n}: n \geq 1\right)$ be an increasing sequence of positive operators in $\mathfrak{A}$ such that

$$
\sup _{n \geq 1} \phi\left(A_{n}^{2}\right)<\infty .
$$

Does there exist a vector $\xi$ in $L_{2}(\mathfrak{A}, \phi)$ such that

$$
\pi\left(A_{n}\right) \omega \stackrel{b}{\rightarrow} \xi \text { and }\left\|\pi\left(A_{n}\right) \omega-\xi\right\| \rightarrow 0 \quad \text { as } \quad n \rightarrow \infty ?
$$

In [6. Theorem 1] we proved that the answer to Problem 10is yes, however under very restrictive additional conditions. In the general case the problem has remained open. In this paper, we exhibit a counterexample revealing that the answer to Problem 1 lies in the negative. More precisely, we shall prove the following

Theorem 1. Given any separable complex Hilbert space $H$, there exist a faithful, normal state $\phi$ on $\mathcal{L}(H)$ and an increasing sequence $\left(A_{n}: n \geq 1\right)$ in $\mathcal{L}(H)_{+}$such that (2.1) is satisfied, but both limit relations in (2.2) fail to be satisfied for any vector $\xi$ in $L_{2}$.

In [7, p. 335] we raised the following problem.

Problem 2. Let $\left(A_{n}: n \geq 1\right)$ be an increasing sequence of operators in $\mathfrak{A}_{+}$such that for some subsequence $\left(A_{n_{k}}: 1 \leq n_{1}<n_{2}<\cdots\right)$ and some vector $\xi$ in $L_{2}$, we have

$$
\pi\left(A_{n_{k}}\right) \omega \stackrel{b}{\rightarrow} \xi \text { and }\left\|\pi\left(A_{n_{k}}\right) \omega-\xi\right\| \rightarrow 0 \quad \text { as } \quad k \rightarrow \infty .
$$

Is it then true that for the whole sequence $\left(A_{n}: n \geq 1\right)$ both limit relations in (2.2) are also satisfied?

Our construction in the proof of Theorem 1 makes it plausible that the answer to Problem 2 also lies in the negative. In fact, we shall prove the following

Theorem 2. Given any separable complex Hilbert space $H$, there exist a faithful, normal state $\phi$ on $\mathcal{L}(H)$ and an increasing sequence $\left(A_{n}: n \geq 1\right)$ in $\mathcal{L}(H)_{+}$such that (2.1) and both limit relations in (2.3) are satisfied for $n_{k}:=2 k, k \geq 1$, but both limit relations in (2.2) fail to be satisfied for any vector $\xi$ in $L_{2}$.

\section{Auxiliary RESUlts}

Being the Hilbert space $H$ separable in our Theorems 1 and 2, we fix an orthonormal basis $\left(e_{k}: k \geq 1\right)$ in $H$. For the reader's convenience, we recall two well-known lemmas, which play key roles in the proofs of Theorems 1 and 2.

Lemma 1. Let $\mathfrak{A}$ be a von Neumann algebra in $\mathcal{L}(H)$ and let $\left(\varepsilon_{k}: k \geq 1\right)$ be a sequence of positive real numbers such that

$$
\sum_{k=1}^{\infty} \varepsilon_{k}^{2}=1
$$


Then

$$
\phi(A):=\sum_{k=1}^{\infty} \varepsilon_{k}^{2}\left(A e_{k} \mid e_{k}\right), \quad A \in \mathfrak{A},
$$

is a faithful, normal state on $\mathfrak{A}$.

In fact, $\phi$ is clearly a positive linear functional on $\mathfrak{A}, \phi(1)=1$, and $\phi$ is faithful (due to the assumption that each $\varepsilon_{k}>0$ ). The normality of $\phi$ is a consequence of [1, Théorème 1 on p. 51]. (See also [3, (A.32) Theorem on p. 121].)

Lemma 2. Let $M$ be $a 2 \times 2$ matrix of the form

$$
M:=\left[\begin{array}{ll}
a & c \\
\bar{c} & b
\end{array}\right], \quad \text { where } \quad a \geq 0, b \geq 0, \text { and } c \in \mathbb{C} .
$$

Then $M$ is positive (semidefinite) if and only if $|c|^{2} \leq a b$.

In fact, the eigenvalues $\lambda_{1}$ and $\lambda_{2}$ of $M$ are given by

$$
\lambda_{1,2}=\frac{1}{2}\left(a+b \pm \sqrt{(a-b)^{2}+4|c|^{2}}\right),
$$

and the one corresponding to the "-" sign in front of the square root is nonnegative exactly in the case when $|c|^{2} \leq a b$.

\section{Proofs of theorems}

Proof of Theorem 1. We shall identify a linear operator $A$ in $\mathcal{L}(H)$ by its (infinite) matrix in the orthonormal basis $\left(e_{k}: k \geq 1\right)$ of $H$. Actually, in our construction we shall consider only such operators $A$ whose matrices contain finitely many nonzero entries. We shall use the same notation for the operator and its matrix in the basis $\left(e_{k}: k \geq 1\right)$. Furthermore, we follow the common agreement that the basis vectors $e_{k}$ are transformed with respect to columns (in accordance with the row-column composition rule when a column vector is multiplied by a matrix from the left).

First, we define a sequence $\left(D_{n}: n \geq 1\right)$ in $\mathcal{L}(H)$ as follows: Set $D_{1}:=O$, the zero operator (or matrix); while for $n \geq 2$,

$$
\left(D_{n}\right)_{1,1}:=2\left(2^{-2}+3^{-2}+\ldots+n^{-2}\right), \quad\left(D_{n}\right)_{n, n}:=n^{2} ;
$$

and for $n \geq 3$,

$$
\left(D_{n}\right)_{j, j}:=j^{2}+(j+1)^{2} \quad \text { for } \quad j=2,3, \ldots, n-1 ;
$$

and all other entries in $D_{n}$ equal 0 . So, each $D_{n}$ for $n \geq 2$, is a diagonal matrix with only nonzero entries in the positions $(1,1)(2,2), \ldots,(n, n)$.

Second, we define another sequence $\left(T_{n}: n \geq 1\right)$ in $\mathcal{L}(H)$ as follows: Set $T_{1}:=O$, while for $n \geq 2$,

$$
\left(T_{n}\right)_{1, n}=\left(T_{n}\right)_{n, 1}:=1,
$$

and all other entries in $T_{n}$ equal 0 . So, each $T_{n}$ for $n \geq 2$ contains only two nonzero entries.

Third, we define

$$
A_{n}:=D_{n}+T_{n}, \quad n \geq 1 .
$$

We shall prove that the sequence $\left(A_{n}: n \geq 1\right)$ and the faithful, normal state $\phi$ defined in (3.2) with (3.1) fulfill the properties stated in Theorem 1 . 
To this effect, we consider the nonzero entries in the difference $A_{n+1}-A_{n}$ for $n \geq 2$ :

$$
\begin{aligned}
\left(A_{n+1}-A_{n}\right)_{1,1} & =2(n+1)^{-2}, \\
\left(A_{n+1}-A_{n}\right)_{1, n} & =\left(A_{n+1}-A_{n}\right)_{n, 1}=-1, \\
\left(A_{n+1}-A_{n}\right)_{1, n+1} & =\left(A_{n+1}-A_{n}\right)_{n+1,1}=1, \\
\left(A_{n+1}-A_{n}\right)_{n, n} & =\left(A_{n+1}-A_{n}\right)_{n+1, n+1}=(n+1)^{2} .
\end{aligned}
$$

It is easy to see that $A_{n+1}-A_{n}$ can be represented as the sum of two matrices $A_{n+1}^{(1)}$ and $A_{n+1}^{(2)}$ (each of them turns out to be positive):

$$
A_{n+1}-A_{n}=A_{n+1}^{(1)}+A_{n+1}^{(2)},
$$

where

$$
\begin{aligned}
\left(A_{n+1}^{(1)}\right)_{1,1} & =\left(A_{n+1}^{(2)}\right)_{1,1}=(n+1)^{-2}, \\
\left(A_{n+1}^{(1)}\right)_{n, n} & =\left(A_{n+1}^{(2)}\right)_{n+1, n+1}=(n+1)^{2}, \\
\left(A_{n+1}^{(1)}\right)_{1, n} & =\left(A_{n+1}^{(1)}\right)_{n, 1}=-1, \\
\left(A_{n+1}^{(2)}\right)_{1, n+1} & =\left(A_{n+1}^{(2)}\right)_{n+1,1}=1,
\end{aligned}
$$

and all other entries in $A_{n+1}^{(1)}$ and $A_{n+1}^{(2)}$, respectively, equal 0. An immediate application of Lemma 2 gives that both $A_{n+1}^{(1)}$ and $A_{n+1}^{(2)}$ are positive operators. By (4.2), the operator $A_{n+1}-A_{n}$ is also positive. Clearly, for $n=1$, the operator $A_{2}-A_{1}=A_{2}$ is also positive.

Next, we shall compute and/or estimate $\phi\left(T_{n}^{2}\right)$ and $\phi\left(D_{n}^{2}\right)$. By the definition of $T_{n}$, it follows from (3.2) that

$$
\phi\left(T_{n}^{2}\right)=\sum_{k=1}^{\infty} \varepsilon_{k}^{2}\left\|T_{n} e_{k}\right\|^{2}=\varepsilon_{1}^{2}\left\|T_{n} e_{1}\right\|^{2}+\varepsilon_{n}^{2}\left\|T_{n} e_{n}\right\|^{2}=\varepsilon_{1}^{2}+\varepsilon_{n}^{2}, n \geq 2 .
$$

By (3.1), we certainly have

$$
\phi\left(T_{n}^{2}\right) \leq 1, \quad n \geq 1\left(T_{1}:=O\right) .
$$

Now, we shall be concerned with $\phi\left(D_{n}^{2}\right)$. To this end, we introduce the notations

$$
\lambda_{k}:=k^{-1} \text { and } B_{k}:=k\left(D_{k+1}-D_{k}\right), \quad k \geq 1 .
$$

Clearly, we have

$$
D_{n+1}=\sum_{k=1}^{n} \lambda_{k} B_{k}, \quad n \geq 1 .
$$

By (3.2) and (4.4), we may write that

$$
\begin{aligned}
\phi\left(B_{k}^{2}\right)= & k^{2} \sum_{j=1}^{\infty} \varepsilon_{j}^{2}\left\|\left(D_{k+1}-D_{k}\right) e_{j}\right\|^{2} \\
= & k^{2}\left\{\left\|\varepsilon_{1}\left(D_{k+1}-D_{k}\right) e_{1}\right\|^{2}+\left\|\varepsilon_{k}\left(D_{k+1}-D_{k}\right) e_{k}\right\|^{2}\right. \\
& \left.\quad+\left\|\varepsilon_{k+1}\left(D_{k+1}-D_{k}\right) e_{k+1}\right\|^{2}\right\} \\
= & k^{2}\left\{4 \varepsilon_{1}^{2}(k+1)^{-4}+\varepsilon_{k}^{2}(k+1)^{4}+\varepsilon_{k+1}^{2}(k+1)^{4}\right\}, \quad k \geq 1 .
\end{aligned}
$$


We fix the sequence $\left(\varepsilon_{k}: k \geq 1\right)$ by setting

$$
\varepsilon_{k}:=2^{-k / 2}, \quad k \geq 1 .
$$

Then condition (3.1) is clearly satisfied, and by (4.6), we also have

$$
\sum_{k=1}^{\infty} \phi\left(B_{k}^{2}\right)<\infty
$$

Owing to (4.5) with $\lambda_{k}:=k^{-1}$ and (4.7), we may apply [7, Theorem 1], which guarantees the existence of a vector $\eta$ in $L_{2}$ such that

$$
\pi\left(D_{n}\right) \omega \stackrel{b}{\rightarrow} \eta \text { and }\left\|\pi\left(D_{n}\right) \omega-\eta\right\| \rightarrow 0 \quad \text { as } \quad n \rightarrow \infty .
$$

By (1.1), we have

$$
\phi\left(D_{n}^{2}\right)=\left\|\pi\left(D_{n}\right) \omega\right\|^{2} .
$$

Thus, it follows from (4.8) that

$$
\sup _{n \geq 1} \phi\left(D_{n}^{2}\right)<\infty .
$$

Making use of [4, Lemma 1.2.1 on p. 4], in the case of (4.1) gives

$$
A_{n}^{2} \leq 2\left(D_{n}^{2}+T_{n}^{2}\right), \quad n \geq 1 .
$$

By virtue of (4.3) and (4.9), we conclude that condition (2.1) is satisfied.

It remains to show that either of the limit relations in (2.2) cannot be satisfied. Assuming the contrary, we shall arrive at a contradiction. To go into detail, assume that the first limit relation in (2.2) is satisfied for some $\xi$ in $L_{2}$. Then, by making use of the additivity property of bundle convergence, it follows from (4.8) that

$$
\pi\left(T_{n}\right) \omega \stackrel{b}{\rightarrow} \xi-\eta, \quad \text { in particular, } \quad \pi\left(T_{n+1}-T_{n}\right) \omega \stackrel{b}{\rightarrow} o \text { as } n \rightarrow \infty,
$$

where "o" is the zero vector in $L^{2}$. According to [2, 3.6. Property on p. 31], the limit relation just obtained is equivalent to the following one:

$$
T_{n+1}-T_{n} \stackrel{b, \mathcal{L}(\mathcal{H})}{\longrightarrow} O \quad \text { as } \quad n \rightarrow \infty .
$$

Taking into account the boundedness of the sequence $\left(T_{n}: n \geq 1\right)$, we may apply [6. Lemma 2] and by (4.10) we obtain

$$
\phi\left(\left(T_{n+1}-T_{n}\right)^{2}\right) \rightarrow 0 \quad \text { as } \quad n \rightarrow \infty .
$$

On the other hand, by (3.2) we find

$$
\begin{aligned}
\phi\left(\left(T_{n+1}-T_{n}\right)^{2}\right) & =\sum_{k=1}^{\infty} \varepsilon_{k}^{2}\left\|\left(T_{n+1}-T_{n}\right) e_{k}\right\|^{2} \\
& \geq \varepsilon_{1}^{2}\left\|\left(T_{n+1}-T_{n}\right) e_{1}\right\|^{2}=2 \varepsilon_{1}^{2}, \quad n \geq 1,
\end{aligned}
$$

which contradicts (4.11). This proves that the first limit relation in (2.2) cannot hold for any vector $\xi$ in $L_{2}$.

Now, let us assume that the second limit relation in (2.2) is satisfied for some vector $\xi$ in $L_{2}$. Then, it follows from (4.8) that

$$
\left\|\pi\left(T_{n}\right) \omega-(\xi-\eta)\right\| \rightarrow 0, \quad \text { a fortiori, } \quad\left\|\pi\left(T_{n+1}-T_{n}\right) \omega\right\| \rightarrow 0 \quad \text { as } \quad n \rightarrow \infty .
$$


By (1.1), the latter limit relation is equivalent to (4.11), which again contradicts (4.12). This proves that the second limit relation in (2.2) cannot hold for any vector $\xi$ in $L_{2}$.

The proof of Theorem 1 is complete.

Proof of Theorem 2. It can be done by a slight reorganization of the proof of Theorem 1 as follows. Again, we construct the operators $D_{n}, T_{n}$, and $A_{n}$, and then consider the sequence

$$
A_{1}, D_{2}, A_{3}, D_{4}, A_{5}, D_{6}, \ldots
$$

By (4.1) and (4.2), we may write that

$$
\begin{aligned}
& D_{2 k}-A_{2 k-1}=\left(A_{2 k}-A_{2 k-1}\right)-T_{2 k}=A_{2 k}^{(1)}+\left(A_{2 k}^{(2)}-T_{2 k}\right), \\
& A_{2 k+1}-D_{2 k}=\left(A_{2 k+1}-A_{2 k}\right)+T_{2 k}=\left(A_{2 k+1}^{(1)}+T_{2 k}\right)+A_{2 k+1}^{(2)} .
\end{aligned}
$$

Making use of the positivity of the operators $A_{n}^{(1)}, A_{n}^{(2)}, A_{2 k}^{(2)}-T_{2 k}$, and $A_{2 k+1}^{(1)}+T_{2 k}$ (observe that both $A_{2 k}^{(2)}-T_{2 k}$ and $A_{2 k+1}^{(1)}+T_{2 k}$ are diagonal matrices with only two positive entries), we conclude that the sequence given in (4.13) is indeed an increasing sequence of positive operators.

By (2.1) and (4.9), we have

$$
\sup _{k \geq 1}\left\{\phi\left(A_{2 k-1}^{2}\right), \phi\left(D_{2 k}^{2}\right)\right\}<\infty .
$$

According to (4.8), the subsequence $D_{2}, D_{4}, D_{6}, \ldots$ of (4.13) converges both in $L_{2}$-norm and bundlewise.

On the other hand, analogously to (4.12) we have

$$
\phi\left(\left(T_{2 k+1}-T_{2 k-1}\right)^{2}\right) \geq 2 \varepsilon_{1}^{2}, \quad k \geq 1 .
$$

Now, if the whole sequence in (4.13) were convergent either bundlewise or in $L_{2}$ norm, then we would necessarily have that

$$
\phi\left(\left(T_{2 k+1}-T_{2 k-1}\right)^{2}\right) \rightarrow 0 \quad \text { as } \quad k \rightarrow \infty
$$

(cf. (4.11)). Clearly, (4.14) and 4.15) contradict each other. Thus, the whole sequence in 4.13) cannot be convergent either bundlewise or in $L_{2}$-norm.

This completes the proof of Theorem 2

\section{Concluding Remarks}

(i) In the very particular choice of the faithful, normal state $\phi$ given in (3.2) with (3.1), we are able to present a transparent 'description' of the completion space $L_{2}=L_{2}(\mathfrak{A}, \phi)$. In fact, let $\mathcal{H}$ be the direct Hilbert sum of a countable number of copies of $H$; that is, the elements of $\mathcal{H}$ are all the sequences $\xi=\left(x_{1}, x_{2}, \ldots\right)$ of vectors with components $x_{k}$ in $H$ such that $\sum_{k=1}^{\infty}\left\|x_{k}\right\|_{H}^{2}<\infty$, where $\|\cdot\|_{H}$ denotes the norm in $H$.

Given any operator $A \in \mathfrak{A}$, we define the corresponding operator $\pi(A)$ acting on $\mathcal{H}$ by amplification:

$$
\pi(A) \xi:=\left(A x_{1}, A x_{2}, \ldots\right), \quad A \in \mathfrak{A}, \xi \in \mathcal{H} .
$$

It is routine to check that this mapping $\pi$ is a one-to-one $*$-homomorphism of $\mathfrak{A}$ into the algebra $\mathcal{L}(\mathcal{H})$. 
Now, let

$$
\omega:=\left(\varepsilon_{1} e_{1}, \varepsilon_{2} e_{2}, \ldots\right) \quad \text { and } \quad \mathcal{E}:=\{\pi(A) \omega: A \in \mathfrak{A}\},
$$

where $\left(\varepsilon_{k}: k \geq 1\right)$ is a sequence of positive numbers satisfying condition (3.1), and $\left(e_{k}: k \geq 1\right)$ is an orthonormal basis in $H$. Clearly, $\|\omega\|=1$ and $\mathcal{E}$ is invariant under the action of $\pi(A), A \in \mathfrak{A}$. Since the same is true for the closure $\overline{\mathcal{E}}$ of $\mathcal{E}$ in $\mathcal{H}$, it follows that $\pi$ acts as a $*$-representation of $\mathfrak{A}$ in $\overline{\mathcal{E}}$. Moreover, the vector $\omega$ is cyclic and separating for this action of $\mathfrak{A}$ in $\overline{\mathcal{E}}$. As a consequence, we may identify $L_{2}(\mathfrak{A}, \phi)$ with $\overline{\mathcal{E}}$.

(ii) We recall that Kadison [5, Proposition 7] proved that if $\left(A_{n}: n \geq 1\right)$ is an increasing sequence of positive operators in a von Neumann algebra $\mathfrak{A}$ such that condition (2.1) is satisfied, then there exists a positive operator $G$ acting on $L_{2}=L_{2}(\mathfrak{A}, \phi)$, affiliated with $\pi(\mathfrak{A})$, with a dense domain $\pi(\mathfrak{A})^{\prime} \omega$ in $L_{2}$, where $\pi(\mathfrak{A})^{\prime}$ means the commutant of $\pi(\mathfrak{A})$, such that

$$
\pi\left(A_{n}\right) T^{\prime} \omega \rightarrow G T^{\prime} \omega \quad \text { weakly as } \quad n \rightarrow \infty,
$$

for each $T^{\prime}$ in $\pi(\mathfrak{A})^{\prime}$. In particular, if $T^{\prime}$ is the identity operator on $L_{2}$, then we have

$$
\pi\left(A_{n}\right) \omega \rightarrow G \omega \quad \text { weakly as } \quad n \rightarrow \infty .
$$

Now, consider our particular sequence $\left(A_{n}: n \geq 1\right)$ of operators introduced in the proof of Theorem 1 by (4.1). By (5.1), we see that

$$
\pi\left(T_{n}\right) \omega=\left(\varepsilon_{1} \varepsilon_{n}, o, \ldots, o, \varepsilon_{n} e_{1}, o, \ldots\right),
$$

whence it follows that for every vector $\xi=\left(x_{1}, x_{2}, \ldots\right)$ in $L_{2}$, we have

$$
\left(\pi\left(T_{n}\right) \omega \mid \xi\right)=\varepsilon_{1}\left(e_{n} \mid x_{1}\right)+\varepsilon_{n}\left(e_{1} \mid x_{n}\right) .
$$

Since

$$
\left(e_{n} \mid x_{1}\right) \rightarrow 0 \text { as } n \rightarrow \infty \quad \text { and } \quad\left|\left(e_{1} \mid x_{n}\right)\right| \leq\left\|x_{n}\right\|_{H} \leq\|\xi\|, \quad n \geq 1,
$$

we conclude that

$$
\left(\pi\left(T_{n}\right) \omega \mid \xi\right) \rightarrow 0 \quad \text { as } \quad n \rightarrow \infty
$$

Or in other words,

$$
\pi\left(T_{n}\right) \omega \rightarrow 0 \quad \text { weakly as } \quad n \rightarrow \infty .
$$

It follows from (4.1), (5.2) and (5.3) that

$$
\pi\left(D_{n}\right) \omega=\pi\left(A_{n}\right) \omega-\pi\left(T_{n}\right) \omega \rightarrow G \omega \quad \text { weakly as } \quad n \rightarrow \infty .
$$

Comparing this with (4.8), we find that $G \omega$ coincides in fact with the strong limit $\eta$ of the sequence $\left(\pi\left(D_{n}\right) \omega: n \geq 1\right)$ in $L_{2}$.

(iii) On closing, we raise the following

Problem 3. Let $\left(A_{n}: n \geq 1\right)$ be an increasing sequence of positive operators in $\mathfrak{A}$ for which there exists a vector $\xi$ in $L_{2}(\mathfrak{A}, \phi)$ such that the second limit relation in $(2.2)$ is satisfied. Is it true or false that then the first limit relation in (2.2) is also satisfied?

Observe that in this case condition (2.1) is automatically satisfied, due to (1.1). 


\section{REFERENCES}

[1] J. Dixmier, Les algèbres d'opérateurs dans l'espace Hilbertien (Algèbres de von Neumann), Gauthier-Villars, Paris, 1969. MR 0352996 (50:5482)

[2] E. Hensz, R. Jajte and A. Paszkiewicz, The bundle convergence in von Neumann algebras and their $L_{2}$-spaces, Studia Math., 120, 1996, 23-46. MR.1398171 (97d:46069)

[3] R. Jajte, Strong limit theorems in noncommutative probability, Lecture Notes in Math., 1110, Springer, Berlin-Heidelberg-New York-Tokyo, 1985. MR0778724 (86e:46058)

[4] R. Jajte, Strong limit theorems in noncommutative $L_{2}$-spaces, Lecture Notes in Math., 1477, Springer, Berlin-Heidelberg-New York, 1991. MF 1122589 (92h:46091)

[5] R. V. Kadison, Some notes on non-commutative analysis, London Math. Soc. Lecture Note Ser., 138, 1989, 243-257. MR1009193 (90i:46114)

[6] B. Le Gac and F. Móricz, Beppo Levi and Lebesgue type theorems for bundle convergence in noncommutative $L_{2}$-spaces, Operator Theory: Advances and Applications, Birkhäuser, 127, 2001, 447-464. MR1902816 (2003g:46073)

[7] B. Le Gac and F. Móricz, Bundle convergence of weighted sums of operators in noncommutative L2-spaces, Bull. Polish Acad. Sci. Math., 49, 2001, 327-336. MR.1872666 (2003d:46085)

[8] M. Takesaki, Theory of operator algebras I, Springer, New York-Heidelberg-Berlin, 1979. MR0548728 (81e:46038)

Université de Provence, Centre de Mathématiques et Informatique, 39 rue JoliotCurie, 13453 Marseille Cedex 13, France

E-mail address: legac@cmi.univ-mrs.fr

Bolyai Institute, University of Szeged, Aradi vértanúk tere 1, H-6720 Szeged, HunGARY

E-mail address: moricz@math.u-szeged.hu 